\title{
E-Band 76-GHz Coherent RoF Backhaul Link Using an Integrated Photonic
} Mixer

Chuenchom, Rattana; Zhou, Xihua; Schrinski, Nils; Babiel, Sebastian; Freire Hermelo, Maria; Steeg, Matthias; Steffan, Andreas; Honecker, Jörg; Leiba, Yigal; Stöhr, Andreas

This text is provided by DuEPublico, the central repository of the University Duisburg-Essen.

This version of the e-publication may differ from a potential published print or online version.

DOI: https://doi.org/10.1109/JLT.2016.2573047

URN: urn:nbn:de:hbz:464-20180620-075923-0

Link: http://duepublico.uni-duisburg-essen.de/servlets/DocumentServlet?id=46401

Legal notice:

(C) 2016 IEEE. Personal use of this material is permitted. Permission from IEEE must be obtained for all other uses, in any current or future media, including reprinting/republishing this material for advertising or promotional purposes, creating new collective works, for resale or redistribution to servers or lists, or reuse of any copyrighted component of this work in other works

Source: This is the author's version of an article that has been published in "Journal of Lightwave Technology, 2016, Vol. 34, No. 20"; Changes were made to this version by the publisher prior to publication.

The final version of record is available at https://doi.org/10.1109/JLT.2016.2573047 


\title{
E-Band 76 GHz Coherent RoF Backhaul Link Using An Integrated Photonic Mixer
}

\author{
Rattana Chuenchom, Xihua Zou, Member, IEEE, Nils Schrinski, Sebastian Babiel, \\ Maria Freire Hermelo, Matthias Steeg, Andreas Steffan, Member, IEEE, Jörg Honecker, Yigal Leiba, \\ and Andreas Stöhr, Senior Member, IEEE
}

(Invited Paper)

\begin{abstract}
An E-band 76 GHz coherent radio-over-fiber (CRoF) system has been developed employing an integrated coherent photonic mixer (CPX) in the remote access unit (RAU) and a Schottky envelope detector in the wireless receiver. The CPX basically consists of a $2 \times 2$ multi-mode interferometer (MMI) coupled to a balanced photodiode (PD). It enables direct conversion of the optical baseband signal to the wireless RF signal with a carrier frequency at $76 \mathrm{GHz}$ using a local oscillator located in the RAU. The developed CPX module features a V-type connector. The $3 \mathrm{~dB}$ cut-off frequency and $1 \mathrm{~dB}$ saturation output power of the CPX module are estimated as $\sim 70 \mathrm{GHz}$ and -2.43 $\mathrm{dBm}$, respectively. It is shown that due to the integration of the MMI and the balanced PD, the developed CPX outperforms a commercial $110 \mathrm{GHz}$ photodiode in terms of conversion efficiency up to $92 \mathrm{GHz}$. Experimentally, long-distance wireless transmission is shown using the constructed CRoF system and highly directive antennas with a gain of $43 \mathrm{dBi}$ each. A wireless transmission of $1 \mathrm{Gbit} / \mathrm{s} \mathrm{NRZ}$ data signal at $76 \mathrm{GHz}$ carrier frequency is demonstrated up to $230 \mathrm{~m}$. The receiver sensitivity of the constructed wireless receiver for a pre-FEC BER of $10^{-3}$ has been measured to be $-34.8 \mathrm{dBm}$. This has enabled wireless data transmission over $92 \mathrm{~m}$ and $230 \mathrm{~m}$ at transmit power levels as low as $-11.17 \mathrm{dBm}$ and $-3.36 \mathrm{dBm}$, respectively. It is shown that the experimental transmit power levels agree well with the expected figures calculated using an analytic Friis model describing the wireless channel. It is also estimated that the system could support maximum wireless distances up to $2000 \mathrm{~m}$.
\end{abstract}

Index Terms-Microwave photonics, heterodyning, photodetectors, fiber optics systems, optical communications, wireless communications, coherent photonic systems

Manuscript received January, 2016. The work was in part supported by the European Project (IPHOBAC-NG) and the EU-Japan research project (RAPID5G). R. Chuenchom was financially support by the Royal Thai Government. X. Zou was supported by the Alexander von Humboldt Foundation, Germany.

R. Chuenchom, N. Schrinski, S. Babiel, M. Freire, M. Steeg and A. Stöhr are with the Institute of Optoelectronics, University of Duisburg-Essen, 47057 Duisburg, Germany. (email: andreas.stoehr@uni-due.de)

$\mathrm{X}$. Zou is with the Center for Information Photonics and Communications, School of Information Science and Technology, Southwest Jiaotong University, Chengdu 611756, China. He is also a Humboldt Research Fellow in the Institute of Optoelectronics, University of Duisburg-Essen, 47057 Duisburg, Germany. (email: zouxihua@swjtu.edu.cn)

A.G. Steffan and J. Honecker are with Finisar, Reuchlinstr. 10/11, 10553 Berlin, Germany. (email: andreas.steffan@finisar.com)

Y. Leiba is with the Siklu Communications Ltd., Asivim 43, Petach-Tikva, Israel (email: yigal@siklu.com)

\section{INTRODUCTION}

$1 \mathbf{V}^{\mathrm{O}}$ OWADAYS wireless communications are indispensable for civil and military sectors. In particular, the demand for high data rate or high capacity wireless communication systems has grown exponentially within the last years. For instance, it is predicted that the monthly global mobile data traffic will surpass 24.3 Exabyte by 2019 [1]. For providing such enormous capacities, it will be necessary to develop wireless systems operating at millimeter-wave bands and even terahertz bands [2]-[13] because international regulatory bodies have opened up more bandwidth for wireless services in these frequency ranges as compared to the microwave region. One of the most interesting frequency bands for medium and long range backhaul links is the E-band, where the $71-76 \mathrm{GHz}$ and 81-86 GHz frequency regions can be used for wireless services. For the E-band, the atmospheric absorption coefficient is substantially smaller than that in other millimeter-wave bands such as the 57-64 GHz band [14]. It is only between 0.35-0.46 $\mathrm{dB} / \mathrm{km}$ for the frequency range of $71-76 \mathrm{GHz}$ and about $0.35 \mathrm{~dB} / \mathrm{km}$ for the $81-86 \mathrm{GHz}$ band.

For providing the predicted huge wireless fronthaul capacity required e.g. in future 5G mobile systems, the mobile backhaul link remains being a bottleneck for system developments. To overcome this limitation, a coherent radio-over-fiber (CRoF) approach has been proposed providing wireless backhauling [15]-[16] and interfacing the radio access units (RAU) in future $5 \mathrm{G}$ mobile networks with high-capacity next generation passive optical networks (PONs) [17]-[18]. In the CRoF architecture, the high-speed mobile data is carried via a fiber optic link to the remotely distributed RAUs. There, the optical data signal gets directly converted to the desired millimeter-wave signal (direct optic-to-RF conversion) by optical heterodyne up-conversion using a free-running, unlocked local oscillator (LO) laser located in the RAU. In conventional RoF systems there is an associated optical loss of $3 \mathrm{~dB}$ (i.e. an RF loss of $6 \mathrm{~dB}$ ) when combining the optical data signal with the optical LO signal in the RAU using a conventional $3 \mathrm{~dB}$ coupler and a conventional photodetector (PD) with a single optical input. This leads to a high conversion loss and thus a poor energy efficiency. To overcome this problem, we have developed a novel integrated coherent photonic mixer (CPX) consisting of a $2 \times 2$ multi-mode interferometer (MMI) connected to a balanced PD [17]. 
In this paper, we report on a field trial demonstrating long-distance $71-76 \mathrm{GHz}$ millimeter-wave $\mathrm{CRoF}$ wireless transmission employing an integrated and packaged broadband CPX in the RAU for direct optic-to-RF conversion. First, it is theoretically shown that the CPX could provide up to $6 \mathrm{~dB}$ higher conversion efficiency as compared to the combination of a $3 \mathrm{~dB}$ coupler and a conventional PD for the same optical LO power. Next, the developed integrated CPX is introduced and experimentally characterized with respect to its RF responsivity and RF output power. The CPX's response is also compared to a commercial $110 \mathrm{GHz}$ PD (Finisar XPDV412xR) for reference showing a substantial improvement in conversion efficiency. Next, long-distance E-band $76 \mathrm{GHz}$ wireless transmission employing the developed CPX and an optical LO laser in the RAU is experimentally investigated in an outdoor field trial. We successfully demonstrate real-time $1 \mathrm{Gbit} / \mathrm{s}$ wireless data transmission at $76 \mathrm{GHz}$ over $92 \mathrm{~m}$ and $230 \mathrm{~m}$. The sensitivity of the developed wireless receiver has been experimentally determined to be as low as $-34.8 \mathrm{dBm}$ for a pre-FEC receiver bit error rate (BER) of $10^{-3}$. By comparing the experimental results with a theoretical link model, it is confirmed that the developed CRoF backhaul would support wireless distances up to $2000 \mathrm{~m}$ when being operated at the maximum available transmit RF power level.

\section{Coherent Photonic Mixer}

As shown in [15], a CRoF system using an envelope detector in the wireless receiver provides robustness against laser phase noise and RF carrier drift. This allows to remotely locate the LO laser in the RAU since phase-locking between the optical signal and the optical LO lasers is not necessary. Recently, CRoF system experiments have proven that although the signal laser in the central station and the LO laser in the remote RAU are unlocked, the CRoF system architecture is highly linear and even allows the development of CRoF systems with record modulation efficiencies up to 9 bit/symbol (512 QAM-OFDM) at $60 \mathrm{GHz}$ [19].

In order to efficiently multiplex the optical data signal with the optical LO signal in the remote RAU and to simultaneously perform direct optic-to-RF conversion, we developed an integrated CPX [17]. A conventional analog RoF link where the signal and LO lasers are both located in the central unit suffers from at least $3 \mathrm{~dB}$ optical loss when combining the two lasers with a $3 \mathrm{~dB}$ coupler [20]. In addition, the LO laser signal gets attenuated by the optical fiber link between the central unit and the RAU which further reduces the energy efficiency of the system. The developed CPX basically consists of a $2 \times 2 \mathrm{MMI}$ coupled to a balanced high-frequency photodiode pair as shown in Fig. 1. The InP-based CPX chip features evanescently coupled pin-type waveguide photodiodes in a balanced configuration with an individual cut-off frequency $\left(f_{3 \mathrm{~dB}}\right)$ of $\sim 75$ $\mathrm{GHz}$, an integrated bias-T for each photodiode as well as two parallel $100 \Omega$ impedances between the signal and ground contacts of the electrical RF output coplanar waveguide (CPW). The CPX chip has been integrated using a Kovar package featuring two optical single-mode fiber inputs and a single RF
V-type coaxial output [17]. It should be mentioned at this point that although the V-type coaxial connector is expected to limit the performance of the integrated CPX module for frequencies above $65 \mathrm{GHz}$, it has been chosen because of cost reasons.

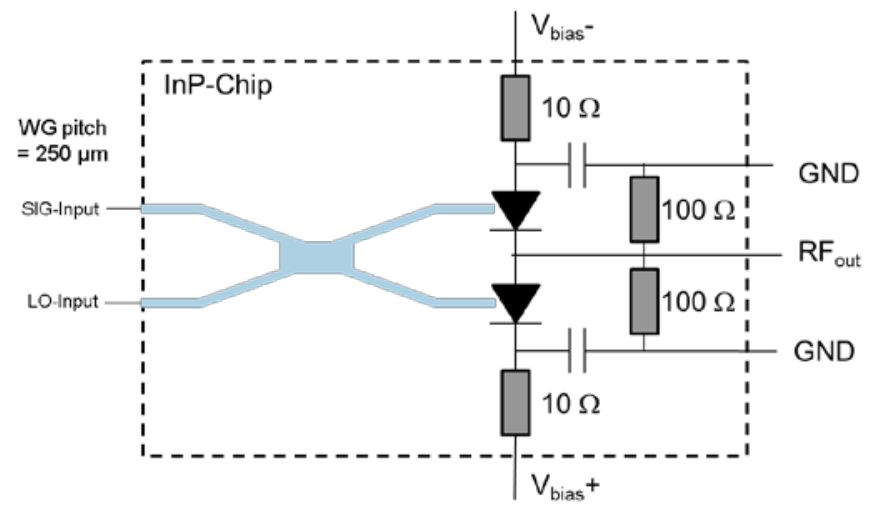

Fig. 1. Schematic of the integrated CPX with the optical signal and the LO laser interfaces on the left and electrical output on the right side [17].

The $2 \times 2$ MMI of the CPX chip has been designed to be symmetrical such that both optical input signals, the optical data signal and the LO signal, are equally 50/50 divided and injected into the balanced photodiode pair. Thus, two photocurrents are generated by optical heterodyning which are equal except for a phase difference of $\pi$. Subtracting those two photocurrents cancels out the DC components whereas the RF photocurrents add up. The total optical power of the two constituent laser signals is completely utilized for performing direct optic-to-RF conversion.

The maximum theoretical improvement of the $\mathrm{RF}$ conversion gain when using the CPX instead of a $3 \mathrm{~dB}$ coupler and a conventional PD can be explained as follows. Assuming that the optical data signal and the LO signal have fixed power levels of $P_{\text {opt,sig }}$ and $P_{\text {opt,Lo, one can calculate the output }}$ photocurrent of a single photodiode when using a $3 \mathrm{~dB}$ coupler to combine the two optical signals from the well-known equation for optical heterodyning

$$
\begin{aligned}
& i_{p h}=\Re \cdot\left(\frac{P_{o p t, s i g}+P_{o p t, L O}}{2}\right)+ \\
& 2 \cdot \Re \cdot \sqrt{\left(\frac{P_{o p t, s i g}}{2} \cdot \frac{P_{o p t, L O}}{2}\right)} \cdot \cos \left(2 \pi f_{R F}+\Delta \emptyset\right),
\end{aligned}
$$

where $\Re$ is the responsivity of a single photodiode, $f_{\mathrm{RF}}$ and $\Delta \phi$ the frequency difference and the phase difference between the two optical signals, respectively. Due to the balanced architecture the DC photocurrent [first term in (1)] is canceled out and only the RF photocurrent [second term in (1)] adds up. Thus the output RF power of the CPX can be written as

$$
P_{R F, C P X} \propto Z\left[i_{p h, R F}-\left(-i_{p h, R F}\right)\right]^{2}=4 Z \Re^{2} \cdot P_{o p t, s i g} \cdot P_{o p t, L O}
$$

where $i_{p h, R F}$ represents the RF photocurrent of a single photodiode, $Z$ is the $50 \Omega$ output impedance. According to (1) the corresponding RF output power of a single PD would be 
proportional to

$$
P_{R F, P D} \propto Z i_{p h, R F}^{2}=Z \Re^{2} P_{o p t, s i g} \cdot P_{o p t, L O}
$$

From (2) and (3), it is clear that the CPX theoretically provides a $6 \mathrm{~dB}$ higher conversion efficiency compared to a conventional PD, assuming identical responsivities and output impedances and an ideal 2×2 MMI.

Besides the higher conversion efficiency, another advantage of locating the LO laser in the remote RAU is that the LO signal is not attenuated by the fiber link. For a $25 \mathrm{~km}$ long fiber link with an attenuation coefficient of $0.2 \mathrm{~dB} / \mathrm{km}$ this would lead to an additional $5 \mathrm{~dB}$ loss. Thus, the $\mathrm{CRoF}$ architecture also enables higher energy efficiency as compared to a conventional analog RoF system [21].

\section{A. RF Responsivity of The Integrated CPX}

First of all, the RF responsivity of the CPX is measured and compared with that of a $110 \mathrm{GHz}$ commercial PD (Finisar XPDV412xR). With the assistance of a heterodyne setup, the LO laser installed in the RAU is tuned to vary the frequency difference between DC and $110 \mathrm{GHz}$. Here, the frequency of the optical signal laser was fixed at $193.54 \mathrm{THz}$. The DC sensitivity of the commercial photodiode and the low-frequency sensitivity of the CPX are $\mathfrak{R}=0.54 \mathrm{~A} / \mathrm{W}$ and $\mathfrak{R}$ $=0.42 \mathrm{~A} / \mathrm{W}$, respectively.

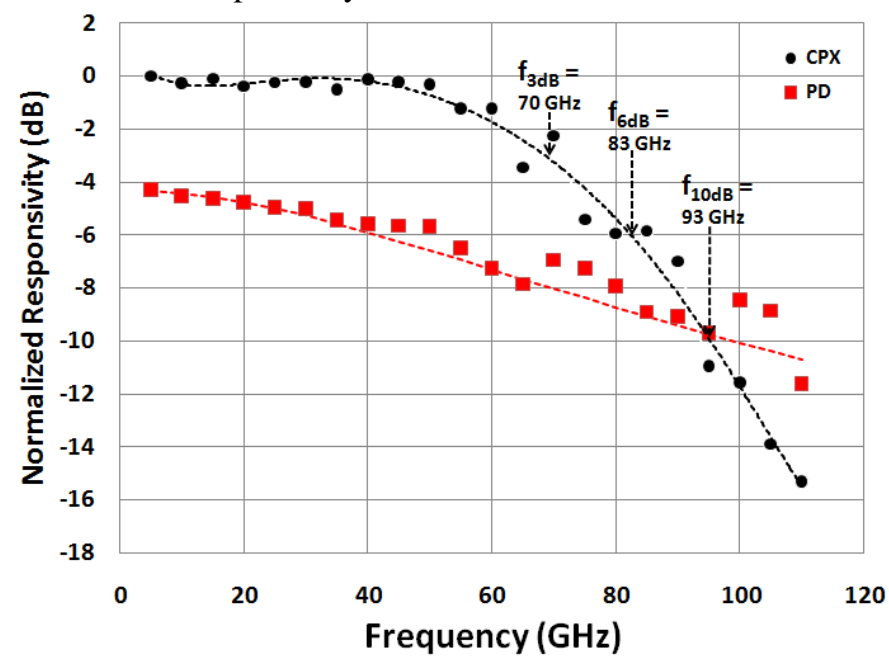

Fig. 2. RF responsivities of the integrated $\mathrm{CPX}$ and the commercial photodetector. The $3 \mathrm{~dB}, 6 \mathrm{~dB}$, and $10 \mathrm{~dB}$ cut-off frequencies are $70 \mathrm{GHz}$, $83 \mathrm{GHz}$, and $93 \mathrm{GHz}$, respectively.

By tuning the frequency of the LO laser, the RF output powers versus frequency were measured for both the CPX and the commercial PD. The output power levels were then normalized to the low-frequency RF output power of the CPX and are shown in Fig. 2. For the CPX, a flat response is observed within the frequency range between DC and $60 \mathrm{GHz}$. A notch close to $67 \mathrm{GHz}$ is observed, which is expected to be due to an impedance mismatch when coupling to the $\mathrm{V}$-type connector. Overall, the RF responsivity of the CPX is about 4-6 dB higher than that of the commercial $\mathrm{PD}$ within the range from DC to $70 \mathrm{GHz}$, indicating a high energy efficiency when converting the optical signal to the RF signal in the RAU.
There is a slight difference of about $1 \mathrm{~dB}$ between the theoretical maximum gain of $6 \mathrm{~dB}$ derived from (2) and (3) and the measured improvement of $5 \mathrm{~dB}$ in average for frequencies below $60 \mathrm{GHz}$. This is expected to be due to the additional RF loss caused by the integrated RF power combiner circuit in the balanced PD.

As can be seen from Fig. 2, the CPX outperforms the commercial $110 \mathrm{GHz}$ photodiode up to about $92 \mathrm{GHz}$ but then its performance drops below the one of the PD. This is mainly because the CPX has been packaged with a V-type coaxial connector with a $3 \mathrm{~dB}$ cut-off frequency of $65 \mathrm{GHz}$ while the commercial PD is packaged with a W1-type connector with a 3 dB cut-off frequency of $110 \mathrm{GHz}$.

\section{B. Output RF Power Level}

Next, the RF output power levels at $76 \mathrm{GHz}$ are measured after the CPX and after the transmitter amplifier chain (see also Fig. 4). Fig. 3(a) shows the RF output power after the CPX versus the photocurrent. At higher photocurrent level some saturation is observed limiting the maximum RF output power from the CPX to $-2.43 \mathrm{dBm}$. The maximum output power after the transmitter amplifier has been measured to be $+17.31 \mathrm{dBm}$. For both measurements, the frequency of the LO laser has been set $76 \mathrm{GHz}$ apart from that of the signal laser and the optical LO power was fixed at $+6.7 \mathrm{dBm}$.
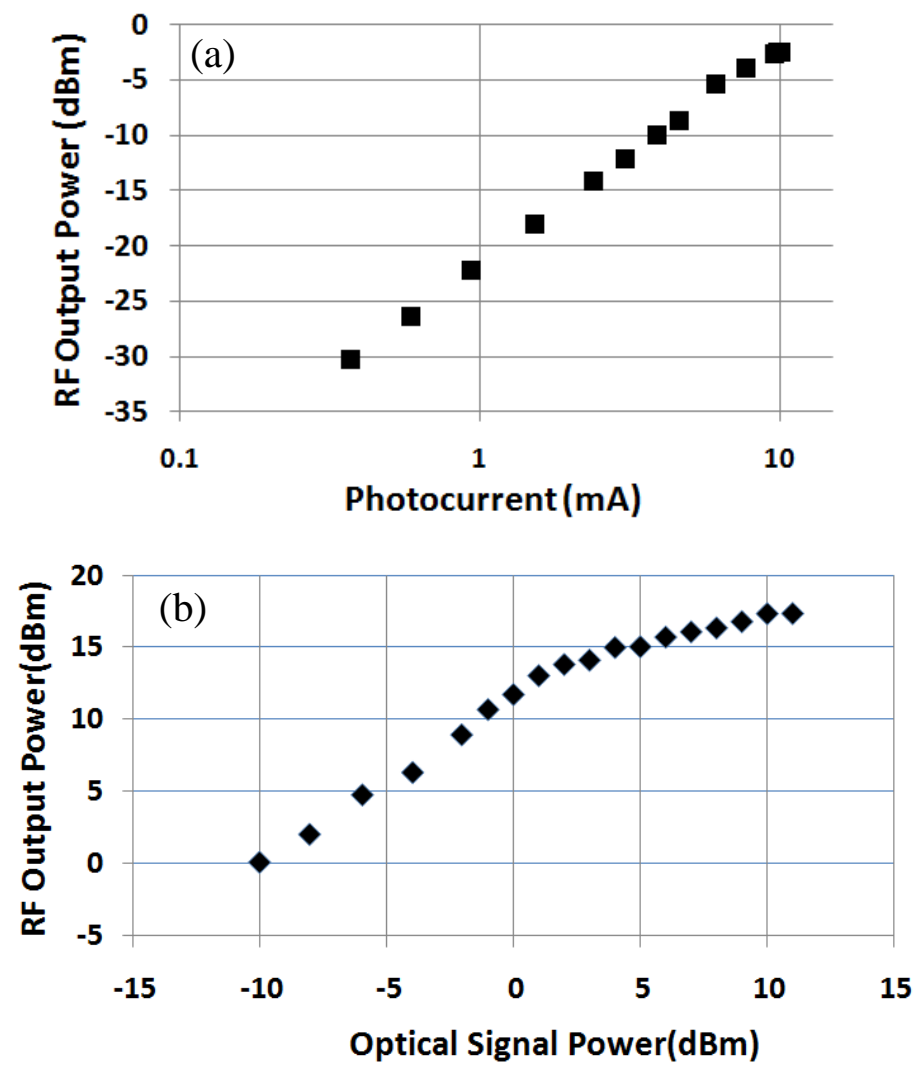

Fig. 3. (a) RF output power from the CPX versus photocurrent and (b) RF output power after the transmitter amplifier chain versus the optical signal power into the CPX at $76 \mathrm{GHz}$ for a fixed optical $\mathrm{LO}$ power of $+6.7 \mathrm{dBm}$. 


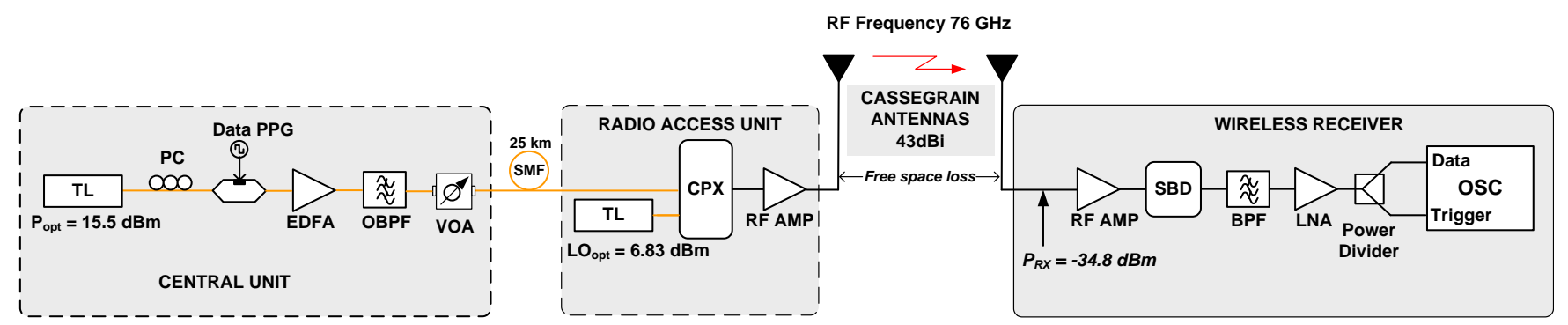

Fig.4. Block diagrams of the CRoF backhaul link using the CPX in the RAU. The wireless signal is received by a Schottky barrier detector (SBD) in the wireless receiver. The eye diagrams were recorded using an oscilloscope (HP54750A).

\section{LONG-DiSTANCE CROF BACKHAUL LINK}

In order to experimentally investigate the performance of the $\mathrm{CPX}$ in a system experiment, a long-distance CRoF wireless backhaul system has been developed using the CPX in the RAU, highly directive antennas and an envelope detector in the wireless receiver.

\section{A. CPX for Outdoor Backhaul Links}

The experimental setup with the CPX in the RAU is shown in Fig. 4. The optical baseband signal is generated in the central unit (CU) using an external cavity laser diode set to $193.5 \mathrm{THz}$ and a Mach-Zehnder modulator (MZM) biased at quadrature point. The pseudorandom binary sequence (PRBS) non-return-to-zero (NRZ) data signal at a rate of $1 \mathrm{Gbit} / \mathrm{s}$ and a word length of $2^{31}-1$ is generated using a pulse pattern generator (PPG). The modulated optical baseband signal is amplified by an Erbium-doped fiber amplifier (EDFA) and filtered using an optical bandpass filter (OBPF) for noise suppression. The optical baseband signal is then attenuated using a variable optical attenuator (VOA) and transmitted via a $25 \mathrm{~km}$ optical single-mode fiber to the remote RAU. In the RAU, the optical baseband signal is directly converted to RF signal using the CPX and an LO laser set to a lasing frequency exactly $76 \mathrm{GHz}$ apart from the frequency of the signal laser. The generated RF signal at $76 \mathrm{GHz}$ is then amplified using a high-power amplifier chain with a total gain of $32 \mathrm{~dB}$ and a saturation output power of $+17.31 \mathrm{dBm}$. The RF signal is then transmitted towards the wireless receiver using a highly-directive (43 dBi) Cassegrain antenna. The wireless signal is detected using the same antenna, amplified using an LNA with a gain of $22 \mathrm{~dB}$ and detected using a Schottky barrier detector (SBD) with a responsivity of approximately 2200 $\mathrm{mV} / \mathrm{W}$. An oscilloscope is used to record the eye diagrams of the down-converted wireless signals.

It should be mentioned that the CRoF system is insensitive against laser phase noise and wavelength drift [15]. Thus it does not require phase locking between the signal laser and the LO laser which allows to remotely locate the LO laser in the RAU. Furthermore, the envelope detection does not require carrier recovery in the wireless receiver which improves the energy efficiency of the system. The CRoF system is scalable, in principle it allows highest spectral efficiencies and a high capacity as shown in [19].

The focus of this work is on experimentally demonstrating long-distance wireless transmission using a CRoF system architecture. Field trials were carried out at different wireless distances of $92 \mathrm{~m}$ and $230 \mathrm{~m}$ between the RAU and the wireless receiver. Here, the $92 \mathrm{~m}$ backhaul link has been established on the campus, while the $230 \mathrm{~m}$ field trial has been carried out on a suburban farm, as shown in Fig. 5.

In order to measure the receiver sensitivity $\left(P_{R X}\right)$, the eye diagram and the SNR were monitored for the $230 \mathrm{~m}$ long wireless link using an oscilloscope while reducing the transmit power level until a BER of $10^{-3}$ was reached. At this point, the received power after the RF amplifier in the wireless receiver was measured to be $-12.8 \mathrm{dBm}$ using a power sensor with a sensitivity level of -35 dBm (Rohde \& Schwarz NRP-Z58). By subtracting the gain of the RF amplifier of $22 \mathrm{~dB}$, the received power was estimated to be $P_{R X}=-34.8 \mathrm{dBm}$, as shown in the Fig. 4.

The corresponding eye diagram is shown in Fig. 6. Some aberrations are present in the eye diagram, especially an overshoot can be observed from Fig. 6. This can be traced back to the high-power amplifier stage in the transmitter amplifier chain. Note that the overshoot is not present in eye measurements at shorter wireless distances of $40 \mathrm{~m}$ that were carried out without the high power amplifier stage [17].

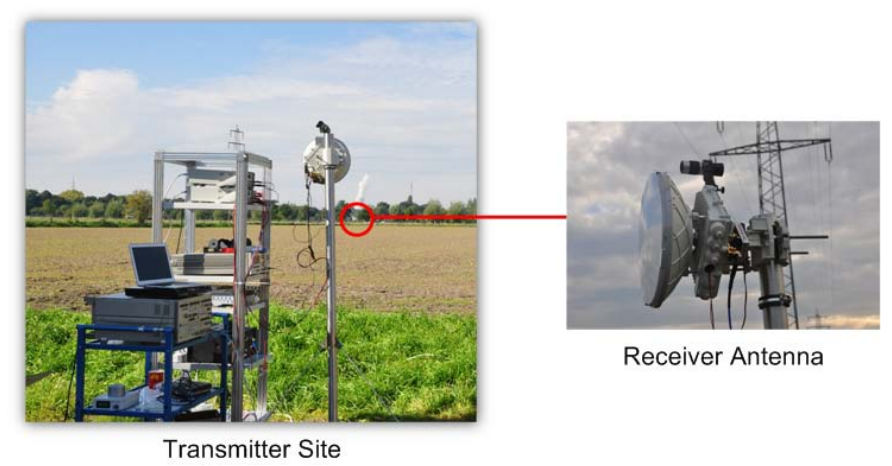

Fig. 5. Photos of the constructed wireless transmitter and receiver units used for the outdoor field trials.

\section{B. Total Path Loss and Maximum Wireless Distance}

In order to determine the maximum achievable wireless distance, the total path loss ( $\left.L_{\text {Path,total }}\right)$ of the backhaul link, which is a combination of the free space propagation loss $\left(L_{\mathrm{P}}\right)$ and the atmospheric absorption attenuation $\left(L_{\mathrm{A}}\right)$, is calculated. The total path loss can be expressed as: 


$$
L_{\text {Path,total }}=L_{P}+L_{A}
$$

As mentioned above, the attenuation coefficient due to atmospheric absorption is below $0.5 \mathrm{~dB} / \mathrm{km}$ at the given $\mathrm{RF}$ carrier frequency and can thus be neglected for the wireless distances under investigation here. The propagation loss can be calculated assuming line-of-sight (LOS) and fair weather conditions in a linear, lossless and isotropic propagation medium [21] from

$$
L_{P}=10 \log _{10}\left(\frac{4 \pi R}{\lambda}\right)^{2}=10 \log _{10}\left(\frac{4 \pi R f}{c}\right)^{2}
$$

where $R$ is the wireless distance, $f$ is the carrier frequency of the RF signal, and $c$ is the light velocity in vacuum. From (4) and (5) and by using the experimentally determined receiver sensitivity $P_{\mathrm{RX}}$, the maximum transmission distance $R_{\max }$ can be derived as

$$
R_{\text {max }}=\frac{c}{4 \pi f} \cdot 10^{\frac{P_{T X}+G_{\text {total }}-P_{R X}}{20}},
$$

where $P_{T X}$ is the transmitted RF power and $G_{\text {total }}$ is the total antenna gain of the link.

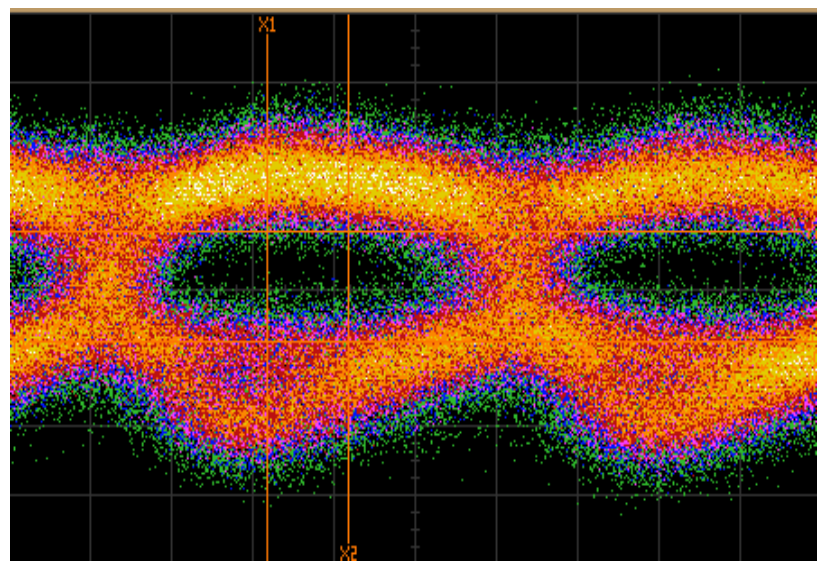

Fig. 6. Eye diagram after wireless transmission over $230 \mathrm{~m}$ for a BER of $10^{-3}$. The received RF power in that case was determined to be $-34.8 \mathrm{dBm}$.

Under the above conditions, the total path loss is calculated to be $108.65 \mathrm{~dB}$ and $116.66 \mathrm{~dB}$ for the $92 \mathrm{~m}$ and $230 \mathrm{~m}$ distance, respectively.

Considering the receiver sensitivity for $\mathrm{BER}=10^{-3}$ which was determined to be $P_{\mathrm{RX}}=-34.8 \mathrm{dBm}$ and the total antenna gain of $G_{\text {total }}=86 \mathrm{~dB}$, the required transmit power can be calculated using (6). Figure 7 shows the required transmit power with respect to the wireless distance for an actual CRoF system under investigation.

In order to compare the actual measurements with the theoretical curve, the transmit power levels were measured after the transmitter amplifier using a power sensor. The measured power levels for $92 \mathrm{~m}$ and $230 \mathrm{~m}$ at a pre-FEC $\mathrm{BER}=10^{-3}$ were $-11.17 \mathrm{dBm}$ or $-3.36 \mathrm{dBm}$, respectively. As can be seen from Fig. 7, the measured transmit power levels agree well with theoretically expected values. Both experimentally determined transmit power levels are about $1 \mathrm{~dB}$ larger than the theoretically expected ones.

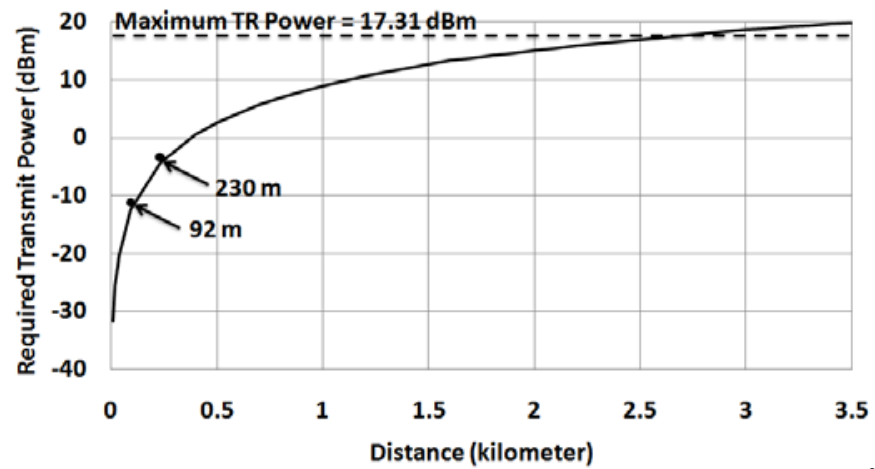

Fig. 7. Theoretically calculated transmit power level for achieving a $B E R=10^{-3}$ with respect to wireless distance. The experimentally determined transmit power levels for $92 \mathrm{~m}$ and $230 \mathrm{~m}$ are also indicated.

It should be pointed out that the maximum wireless distance depends on several system parameters including the antenna gain, antenna alignment, polarization mismatch, amplification gain, weather condition, and so on. However, a first estimation of the maximum achievable wireless distance for the system under investigation can be derived from Fig. 7. Assuming operation at saturation output power of the developed transmitter amplifier chain of $+17.31 \mathrm{dBm}$, the system would support wireless distances beyond $2000 \mathrm{~m}$. This would hold in fair weather conditions. In the case of heavy rain with a precipitation of $25 \mathrm{~mm} / \mathrm{h}$ at $76 \mathrm{GHz}$, the link gain would suffer from an additional $10 \mathrm{~dB} / \mathrm{km}$ attenuation which would then allow the system to be operated up to about $900 \mathrm{~m}$.

\section{CONCLUSION}

An E-band 76 GHz CRoF system using an integrated CPX in the RAU and an envelope detector in the wireless receiver has been demonstrated. Experimentally, $1 \mathrm{Gbit} / \mathrm{s}$ wireless data transmission over $92 \mathrm{~m}$ and $230 \mathrm{~m}$ was demonstrated. The maximum wireless distance the system would support in fair weather condition is estimated to be beyond $2000 \mathrm{~m}$. The constructed CRoF wireless system is insensitive against laser phase noise and carrier frequency drift. Thus it does not require locking between the signal and local oscillator lasers nor does it require any clock recovery in the wireless receiver.

\section{REFERENCES}

[1] —, "Cisco Visual Networking Index: Global Mobile Data Traffic Forecast Update, 2014-2019,” Cisco® Vis. Netw. Index Glob. Mob Data Traffic Forecast Updat., pp. 1-42, 2015.

[2] C. Lim, A. Nirmalathas, D. Novak., R. Waterhouse, and G. Yoffe., "Millimeter-wave broad-band fiber-wireless system incorporating baseband data transmission over fiber and remote LO delivery," $J$. Lightw. Technol., vol. 18, no. 10, pp. 1355-1363, Oct. 2000.

[3] A. M. J. Koonen and M. G. Larrodé, "Radio-over-MMF techniques-part II: microwave to millimeter-wave systems,” $J$. Lightw.Technol., vol. 26, no. 15, pp. 2396-2408, Aug. 2008.

[4] N. J. Gomes, M. Morant, A. Alphones, B. Cabon, J. E. Mitchell, C. Lethien, M. Csörnye, A. Stöhr, and S. lezekiel, "Radio-over-fiber transport for the support of wireless broadband service,” J. Opt. Netw., vol.8, no.2, pp.156-178, Feb. 2009.

[5] J. Yu, G. Chang, Z. Jai, A. Chowdhury, M. Huang, H. Chien, Y. Hsueh, W. Jian, C. Liu, and Z. Dong., "Cost-effective optical millimeter 
technologies and field demonstrations for very high throughput wireless-over-fiber access systems,” J. Lightw. Technol., vol. 28, no.16, pp. 2376-2397, Aug. 2010.

[6] A. Stöhr, S. Babiel, P. J. Cannard, B. Charbonnier, F. van Dijk, S. Fedderwitz, D. Moodie, L. Pavlovic, L. Ponnampalam, C.C. Renaud, D. Rogers, V. Rymanov, A. Seeds, A. G. Steffan, A. Umbach, and M. Weiß, "Millimeter-wave photonic components for broadband wireless systems," IEEE Trans. Microw. Theory Techn., vol. 58, no. 11, pp. 3071-3082, Nov. 2010.

[7] S. Koenig, D. Lopez-Diaz, J. Antes, F. Boes, R. Henneberger, A. Leuther, A. Tessmann, R. Schmogrow, D. Hillerkuss, R. Palmer, T. Zwick, C. Koos, W. Freude, O. Ambacher, J. Leuthold, and I. Kallfass, "Wireless sub-THz communication system with high data rate," Nat. Photonics, vol. 7, no.12, pp. 977-981, Dec. 2013.

[8] A. J. Seeds, H. Shams, M. J. Fice, and C. C. Renaud, "Terahertz photonics for wireless communications,” J. Lightwave Technol., vol. 33, no.3, pp.579-587, Feb. 2015.

[9] T. Nagatsuma, S. Hisatake, M. Fujita, H. H. N. Pham, K. Tsuruda, S. Kuwano, and J. Terada, "Millimeter-wave and terahertz-wave applications enabled by photonics,” IEEE J. Quantum Electron., vol. 52, no. 1, Article\#: 0600912, Jan. 2016.

[10] R. Sambaraju, J. Herrera, J. Marti, D. Zibar, A. Caballero, J. B. Jensen, I. T. Monroy, U. Westergren and A. Walber, "Up to $40 \mathrm{~Gb} / \mathrm{s}$ wireless signal generation and demodulation in $75-110 \mathrm{GHz}$ band using photonic techniques," IEEE International Topic Meeting on Microwave Photonics (MWP 2010), Montreal, Canada, Oct. 2010, Post deadline Paper 1-4.

[11] X. Pang, A. Caballero, A. Dogadaev, V. Arlunno, R. Borkowski, J. S. Pedersen, L. Deng, F. Karinou, F. Roubeau, D. Zibar, X. Yu, and I. T. Monroy, "100 Gbit/s hybrid optical fiber-wireless link in the W-band (75-110 GHz),” Opt Express, vol. 25, no. 19, pp. 24944 -24949, Nov. 2011.

[12] I. S. Amiri, S. E. Alavi, S. M. Idrus, A. S. M. Supa'at, J. Ali, and P. P. Yupapin, "W-Band OFDM transmission for radio-over-fiber link using solitonic millimeter wave generated by MRR,” IEEE J. Quantum Electron., vol. 50, no. 8, pp. 662-628, Aug. 2014.

[13] J. Antes and I. Kallfass, "Performance estimation for broadband multi-gigabit millimeter and sub-millimeter wave wireless communication links," IEEE Trans. Microw. Thoery Techn., vol. 63, no. 10, pp. 3288-3299, Oct. 2015.

[14] ITU-R, "Recommendation ITU-R P.676-10 Attenuation by atmospheric gases", International Telecommunication Union.

[15] A. Stöhr, O. Cojucari, F. van Dijk, G. Carpintero, T. Tekin, S. Formont, I. Flammia, V. Rymanov, B. Khani, and R. Chuenchom, "Robust 71-76 $\mathrm{GHz}$ radio-over-fiber wireless link with high-dynamic range photonic assisted transmitter and laser phase-noise insensitive SBD receiver", Optical Fiber Communication Conference (OFC 2014), Anaheim, United States, March 2014, Paper M2D.4.

[16] M.P. Thakur, S. Mikroulis, C.C. Renaud, J.E. Mitchell, A. Stöhr, "DWDM-PON/mm-wave Wireless Converged Next Generation Access Topology using Coherent Heterodyne Detection,” Int. Conf. on Transparent Opt. Networks (ICTON 2014), Graz, Austria, July 6-10, 2014, Paper Tu.D4.2.

[17] R. Chuenchom, X. Zou, V. Rymanov, B. Khani, M. Steeg, S. Dülme, S. Babiel, J. Honecker, A.G. Steffan and A. Stöhr, "Integrated $110 \mathrm{GHz}$ coherent photonic mixer for CRoF mobile backhaul links" IEEE International Topic Meeting on Microwave Photonics (MWP 2015), Paphos, Cyprus, Oct. 2015, Paper TuC-2.

[18] R. Chuenchom, S. Babiel, M. Steeg and A. Stöhr, "Impact of WDM channel spacing on millimeter-wave wireless access using wireless coherent radio-over-fiber (CRoF) channels" Optical Fiber Communication Conference (OFC 2015), Los Angeles, Unites States, March 2015, Paper TH2A.8.

[19] A. Stöhr, B. Shih, S. T. Abraha, A.G. Steffan, and A. Ng’oma, "High spectral-efficient 512-QAM-OFDM $60 \mathrm{GHz}$ CRoF system using a coherent photonic mixer (CPX) and an RF envelope detector," Optical Fiber Communication Conference (OFC 2016), Anaheim, Unites States, March 2016, Paper Tu3B.4.

[20] J.R Barry and E.A. Lee., "Performance of Coherent Optical Receivers", Pro. IEEE, vol. 78, no. 8, Aug. 1990.

[21] M. Weiß, "60 GHz photonic millimeter-wave communication systems," PhD thesis, University of Duisburg Essen, Germany, 2010
Rattana Chuenchom received the B.S. degree in electronics and computer and M.S. degrees in electrical communication engineering from King Mongkut's Institute of Technology Ladkrabang, Bangkok, Thailand, in 1998 and 2006 respectively.

From 2007 to 2013, she worked with National Institute of Metrology Thailand in Photometry and Radiometry. Since 2013, she has been working towards the Ph.D. degree with the Department of Optoelectronics at the University of Duisburg-Essen. Her current research involves the Photonic Technology Wireless Systems.

Xihua Zou (M'10) received the Ph.D. degree from Southwest Jiaotong University, China, in 2009.

He joined the Center for Information Photonics \& Communications, Southwest Jiaotong University in 2009, where he is currently a full professor. Since October 2014, he has been working as a Humboldt Research Fellow in the Institute of Optoelectronics, University of Duisburg-Essen, Germany. His current interests include microwave photonics, optoelectronic oscillator, radio over fiber, optical pulse generation and compression, and optical communications. He has authored or coauthored over 80 academic papers in refereed journals.

Prof. Zou is an Associate Editor of IEEE Journal of Quantum Electronics, and once was the Editor and the Coordinator for a Focus Issue on Microwave Photonics in IEEE Journal of Quantum Electronics, and the leading Guest Editor for a Special Section on Microwave Photonics in Optical Engineering. He was a recipient of the Alexander von Humboldt Research Fellowship (2014), National Outstanding Expert in Science \& Technology of China (2014), the Nomination Award for the National Excellent Doctoral Dissertation of China (2011), and the Outstanding Reviewer of Optics Communications (2015).

Nils Schrinski received his B.SC in electrical engineering at university Duisburg-Essen in 2015. His Bachelor thesis concentrated on the topic "Theoretic and experimental investigations to long distance point-to-point millimeter-wave link using OOK format and envelope detection SBD”.

He is currently studying M.SC. in electrical engineering with specialization in micro- and optoelectronics at University Duisburg-Essen and is working at the department of Optoelectronics as research assistance.

Sebastian Babiel received his diploma in electrical engineering at university Duisburg-Essen in 2009. Also in 2009 he received an award for an extraordinary diploma thesis with the title "Development and characterization of a photonic 60 $\mathrm{GHz}$ emitter for broadband short- and medium-range communication”. During his studies he worked as student worker for the department of optoelectronics and was involved in the IPHOBAC project where he developed a 6-stage detector array for RF detection for the whole frequency range from $30 \mathrm{GHz}$ to $325 \mathrm{GHz}$.

Since 2010 he is a PhD student working as an early stage researcher in the department of optoelectronics at university Duisburg-Essen working towards $60 \mathrm{GHz}$ RoF system. For indoor and outdoor communications. 
Maria Freire Hermelo received the Telecommunication Engineer degree from the University of Vigo, Vigo, Spain, in 2009 and the M.S. degree in digital signal processing from the same university in 2010.

From 2010 to 2015, she was with Galician Research and Development Center in Advanced Communications. She spent three months as Engineer in Televes. Since 2015, she has been working towards the Ph.D. degree with the Department of Optoelectronics at the University of Duisburg-Essen. Her research interest includes radio-over-fiber systems and signal processing in communications.

Matthias Steeg received his bachelor's and master's degree in electrical engineering and information technology from University Duisburg-Essen in 2012 and 2015, respectively. After his studies with focus on micro- and optoelectronics he joined the Optoelectronics department of University Duisburg-Essen in 2015 as a research assistant. He is currently working towards his Ph.D. under supervision of Prof. Stöhr. His research interests include optical communications, radio-over-fiber, $60 \mathrm{GHz}$ radio, 5G mobile networks and mm-wave antennas.

Andreas Steffan received his Dipl. Ing. and Dipl. Phys. degree from the Rheinisch-Westfälisch Technische Hochschule (RWTH) Aachen. For his thesis he worked on Silicon-on-Insulator (SOI) waveguides. This work was carried out at the Institute of Semiconductor Technology (IHT) in cooperation with AMO GmbH, Aachen. In 2002 he received his Ph.D. from the University of Cambridge, England. In 2002 he joined $\mathrm{u}^{2} \mathrm{t}$ Photonics AG now Finisar. Initially he worked as project manager on the development of new transmitter, receivers and test and measurement products, including modulators and pulsed laser sources. At Finisar he's now heading the $\mathrm{T} \& \mathrm{M}$ / Analogue Products group. He is a member of the Institute of Physics (IoP), and received awards from the Engineering and Physical Science Research Council (EPSRC), Renishaw plc. and the Cambridge European Trust.

Jörg Honecker has been working for Finisar and before $\mathrm{u}^{2} \mathrm{t}$ Photonics AG as Development Engineer since September 2000. He holds the Dipl. Ing after studying 4 years of communication engineering with his main focus on high frequency technology, digital signal handling and transfer mode system. During his study he used to work for Heinrich Hertz Institute on different projects, especially in the field of optical techniques. At FIN
Jörg is in charge of rf-simulation of our devices and all kinds of measurements of high frequencies up to $110 \mathrm{GHz}$. Since his Diploma, Jörg has been continuously focusing on RF measurement techniques with the $67 \mathrm{GHz}$ Lightwave Component Analyzer (LCA) which is similar to a network analyzer with optical interfaces. This makes Jörg our expert in this very important field of measurement techniques.

Yigal Leiba received his B.Sc and M.Sc. degree from the Technion institute of technology in Haifa, Israel. He is currently a co-founder and CTO of Siklu Communication Ltd., specializing in mmwave communication. In previous positions he worked for Runcom Technologies, in charge of the design and the development of the world's first mobile-WiMAX (802.16e) chip, and was a voting member of the IEEE 802.16 standard committee. He was also a co-founder and CTO of Breezecom's LMDS group, a spin-off of Breezecom (later Alvarion (NASDAQ: ALVR)). He also served as chief Engineer in Breezecom, and as an R\&D engineer in the Israeli Ministry of Defense Electronics Research Department.

Andreas Stöhr (M’97) received the Dipl.-Ing. and Dr.-Ing. degrees in Electrical Engineering from Gerhard-Mercator-University, Germany, in 1991 and 1997, respectively. From 1987 until 1996 he was the CEO of MS Steuerungsanlagen GmbH in Germany. From 1996 until 2013 he was a research scientist at University Duisburg. During that period, in 1998 and 1999 he also joined the Communications Research Laboratory (CRL) in Tokyo, Japan where he worked on $60 \mathrm{GHz}$ wireless systems employing radio over fiber techniques. He also worked with France Telecom Orange Labs in Lannion, France in 2009 and with Corning in 2015. Currently, he is also a visiting Professor at the University of Ottawa. Since 2011, he is Professor and head of the Optoelectronics department within the Center for Semiconductor Technology and Optoelectronics (ZHO) at University Duisburg-Essen (UDE), Germany.

His current research interests include III/V integrated microwave photonic device technology and RF photonic integration technologies for millimeter-wave and $\mathrm{THz}$ communications, measurement systems as well as sensing applications. Prof. Stöhr has published more than 200 papers in refereed journals and conferences. He is a senior member in IEEE Photonics and MTT society, committee member and chair of a number of international conferences and IEEE/OSA guest editor. 